Abstract

\title{
Evaluation of the Performance of Permutation Entropy Variants for Classifying Auditory Evoked Potentials ${ }^{+}$
}

\author{
Walter Edgardo Legnani, Marco Gabriel Baldiviezo, Camila Bontempo, Yanina Corsaro, Juan \\ Facundo Fernandez Biancardi, Adrian Paglia, Masia Rebeca Hernando and Matías Rodriguez \\ Signal and Image Processing Center from Universidad Tecnológica Nacional, Buenos Aires, Argentine \\ † Presented at the Entropy 2021: The Scientific Tool of the 21st Century, 5-7 May 2021; Available online: \\ https://sciforum.net/conference/Entropy2021/.
}

Published: 5 May 2021

The aim of the present work was to investigate the performance of entropic tools to classify, subjects with normal hearing and those with pathologies in the auditory pathway, using short-latency records of auditory evoked potentials. To accomplish with this objective, traditional permutation entropy, weighted permutation entropy and a modified version of the original permutation entropy, correcting the count of the missing or forbidden patterns.

The database used consisted of two age groups, one of minors aged from a few months to four years and the other of older aged over 18 years.

For both minor and older subjects, thirty samples were randomly selected from both normal and hearing-impaired subjects.

Once the different varieties of entropy had been calculated, the difference in mean values was analyzed using statistical tests to check whether the difference between them was significant or not. The differences between means of the groups with and without pathology were found to be significantly different at a level of $99.9 \%$.

Finally, the choice of the most appropriate entropy was made based on the calculation of the specificity, selectivity, precision and area under the operation reception curve. The results obtained showed that for the differentiation of healthy and pathological records in the case of minors, the weighted permutation entropy was more appropriate and the modified version of the permutation in the case of older adults.

(C) 2021 by the authors. Licensee MDPI, Basel, Switzerland. This article is an open access article distributed under the terms and conditions of the Creative Commons Attribution (CC BY) license (http://creativecommons.org/licenses/by/4.0/). 